\title{
DETERMINATION OF MAGNETIC CORE PARAMETERS OF ELECTROMAGNETIC ELEMENTS OF FERRO-VORTEX APPARATUS
}

\author{
Andrey Adoshev, Sergey Antonov, Alexander Ivashina, Nikita Garkavenko \\ Stavropol State Agrarian University, Russia \\ adoshev@mail.ru, antonov_serg@mail.ru,av_ivashina49@mail.ru,furfurlis@mail.ru
}

\begin{abstract}
The ferro-vortex apparatus relates to devices of electro-mechanical processing of liquid, bulk and other blends used in agriculture, medicine, chemical, oil and gas industry, communal services and other areas. The energy of the rotating electromagnetic field is concentrated in the working area of the apparatus, which is conditionally limited by the external and internal diameters of the toroidal magnetic cores of electromagnetic elements (inductors) axial shape and the end surface of their tines. Wherein, the value of the working gap in the ferro-vortex apparatus is significantly larger than the air gaps in standard electric machines of both cylindrical and axial execution. The study of the electromagnetic parameters of the apparatus, created on the basis of recommendations received in the process of creating already existing prototypes, revealed a number of shortcomings. At the moment, there is no methodology that allows to design devices with optimal dimensions of magnetic cores of electromagnetic elements. When optimizing their design, it becomes necessary to manufacture new prototypes, which is associated with large time and material costs. An alternative to this is the use of computer technology and appropriate software for mathematical modeling. The article shows a mathematical model representing the dependence of the average diameter of the active surface of magnetic cores and the active length on the electromagnetic loads. When analyzing the model, the optimal ranges for adjusting the size of the working area $(25-35 \mathrm{~mm})$ and the magnitude of the magnetic induction in the air gap were determined $(0.11-0.16 \mathrm{~T})$. On the basis of this model, a technique for designing devices with optimal dimensions of magnetic cores of electromagnetic elements is created.
\end{abstract}

Keywords: ferro-vortex apparatus; magnet core; electromagnetic element; working area.

\section{Introduction}

After conducting studies of the electromagnetic characteristics of the ferro-vortex apparatus (FVA) [1], calculated at a working gap of $\delta=1 \mathrm{~mm}$ and the value of the magnetic induction in it $B_{\delta}=1 \mathrm{~T}$, it became necessary to verify the results obtained.

Experimental studies of FVA as part of the technological line (Fig. 1) showed unsatisfactory results. The temperature of both the winding and the magnetic circuits after several cycles of switching on and off during operation under load began to grow much faster than it was laid down by the technical conditions. This was mainly due to the so-called "human factor".

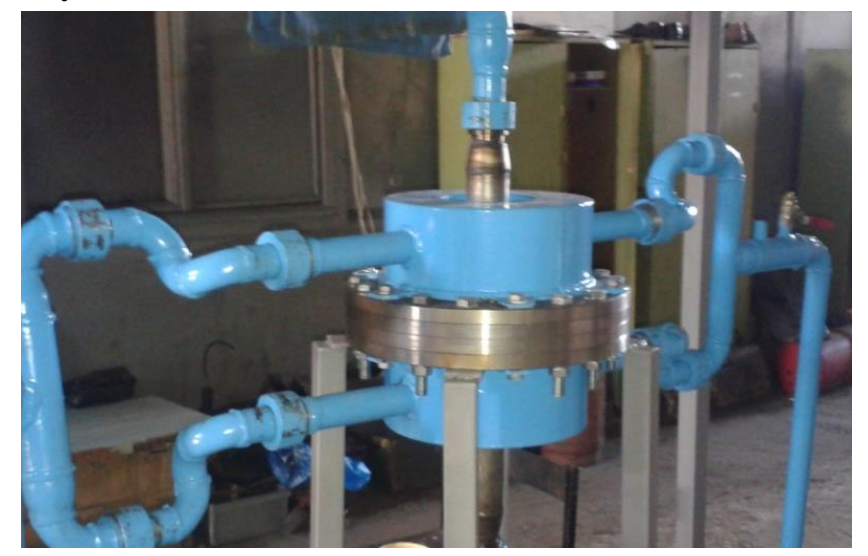

Fig. 1. Ferro-vortex apparatus as part of the technological line

It became necessary to manufacture new magnetic cores of electromagnetic elements of the FVA to study their characteristics, but taking into account the coefficient of induction in the gap [1].

During the manufacturing process, we encountered some difficulties. In particular, it was far from always possible to obtain a core with the necessary overall dimensions. This is due to the fact that the nearest enterprises (manufacturing plants) were not interested in setting up the technological chain for manufacturing small batches of twisted magnetic cores with non-standard sizes of external $\left(D_{\text {out }}\right)$ and 
internal $\left(D_{i n}\right)$ diameters, as well as the width of the electrical steel strip. Therefore, we had to use the existing magnetic cores with standard indicators suitable for us in size.

In this case, the sequence of calculation of the parameters of the electromagnetic elements has changed.

\section{Materials and methods}

The calculation of the apparatus was carried out starting with the determination of the main dimensions - the average diameter of the magnetic core of the electromagnetic element $D_{\text {mid }}$ and the active gap length $l_{\delta}$, which are related to the power $P$, angular velocity $\omega$ and electromagnetic loads and the expression of the machine constant [2-4]:

$$
D_{\text {mid }}^{2} l_{\delta}=\frac{2 P^{\prime}}{\pi \alpha_{\delta} k_{b} k_{o} A B_{\delta} \omega} ;
$$

where $A$-linear load on the average diameter, $\mathrm{A} \cdot \mathrm{m}^{-1}$;

$B_{\delta}$ - magnetic induction in the gap, T;

$\alpha_{\delta}, k_{b}$ and $k_{w}$ - coefficients of flattening, field curve shapes, and winding.

The efficiency of using the volume of the active part of the apparatus is determined by electromagnetic loads: linear load $A$ and induction in the gap $B_{\delta}$. The linear load is determined by the ratio of the current of all the turns of the winding to the circumference. Its value shows what current is on average per unit length of the circumference of the gap of the apparatus. The induction in the gap at the given gap diameter and number of poles determines the magnetic flux of the apparatus and, consequently, the level of induction on the sections of the magnetic circuit [4].

The maximum permissible levels of electromagnetic loads for specific apparatus are determined by the permissible heating of the active parts, since with the growth of $A$ and $B_{\delta}$, the losses in the unit of the active volume of the apparatus increase. The values of electromagnetic loads are set in the form of recommendations in the corresponding calculation methods and serve as the basis for the correct choice of the volume of the active part.

In the initial period, the calculation is carried out by setting the values of electromagnetic loads $(A$ and $B_{\delta}$ ), coefficients $\left(a_{\delta}, k_{b}\right.$ and $\left.k_{w}\right)$, the number of tine $(z)$ and the number of pole pairs $(p)$ based on the available recommendations.

By the known dimensions of the external and internal diameters of the magnetic core, we find the cross-sectional area of its active axial surface $S_{\delta}$ and the active length $\left(l_{\delta}\right)$ of the electromagnetic element of the axial apparatus [2]:

$$
\begin{gathered}
S_{\delta}=\frac{\pi}{4}\left(D_{\text {out }}+D_{\text {in }}\right)\left(D_{\text {out }}-D_{\text {in }}\right) ; \\
l_{\delta}=0.5 \cdot\left(D_{\text {out }}-D_{\text {in }}\right) .
\end{gathered}
$$

Studies have shown that the proportion of the flux of the pole division, the lines of which are closed through the outer and inner layers of the magnetic cores, depends mainly on the gap.

When the gap decreases, the volume of the working area decreases and, accordingly, the mass of ferromagnetic particles in it decreases, because the $K_{c r}$ (critical coefficient-the ratio of the volume of ferromagnetic particles in the working area to the volume of the working area) cannot be exceeded. With an underestimated value of $\delta$, the performance of the apparatus decreases.

With increasing $\delta$, the volume of the working area increases and, accordingly, the mass of ferromagnetic particles in it increases. But, at the same time, an excessive increase in the gap is associated with a decrease in the value of $B_{\delta}$ - one of the main parameters of the magnetic field of the ferro-vortex apparatus. To maintain the recommended values of $B_{\delta}$, with an increase in $\delta$, it is necessary to increase the main dimensions $\left(D_{\text {mid }}\right.$ and $\left.l_{\delta}\right)$, which entails an increase in the mass-dimensional parameters of the apparatus. 
Since in ferro-vortex apparatuses the gap value is much larger than the gaps of standard electric machines of both cylindrical and axial designs, the calculated gap length increases in comparison with the actual one due to this part of the flow.

Thus, the estimated gap length is determined by the formula:

$$
l_{\delta \text {.caic }}=l_{\delta}+2 \delta,
$$

where $l_{\delta}$ - (in this case) the actual active gap length.

In this case the area of the tooth division $\left(S_{t l}\right)$ of the electromagnetic element of the FVA:

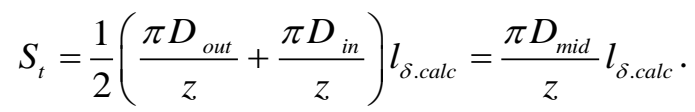

The experience of implementing technological processes in vortex layer apparatuses [5-9] allowed us to determine the appropriate induction interval from 0.1 to $0.2 \mathrm{~T}$. For most apparatuses intended for the production of emulsions, suspensions, and various chemical processes, VLA with an induction of $0.1-0.15 \mathrm{~T}$ is used. Therefore, the required (actual) value of the magnetic induction in the gap $\left(B_{\delta . f a c t}\right)$ is the value that determines the efficiency of the ferro-vortex apparatus, and the calculated value of the magnetic induction in the air gap in $B_{\delta . c a l c}$ is used in the calculations of the FVA calculation, T [1]:

$$
B_{\delta . c a l c}=\frac{B_{\delta . \text { fact }}}{k_{B_{\delta}}},
$$

where $B_{\delta . f a c t}-$ required actual value of the induction in the gap, T;

$k_{B \delta}$ - coefficient of induction in the gap, depending on the size of the gap (selected from the area bounded by the curves constructed on the basis of experiments [1]).

It should be pointed out that the presented dependences are calculated under the condition of optimal basic dimensions. I.e., for each combination of inductions in the sections of the magnetic circuit $\left(B_{z}, B_{a}\right.$ - the average values of induction in the tine and yoke (respectively) of the magnetic core of the electromagnetic element), the corresponding optimal basic dimensions and optimal induction in the air gap were calculated, only after which the corresponding magnetic flux was determined [10].

Magnetic flux of the ferro-vortex apparatus in the air gap:

$$
\Phi_{\delta}=\frac{\alpha_{\delta} S_{\delta}}{2 p} B_{\delta \text {.calc }},
$$

where $B_{\delta . c a l c}-$ calculated value of the magnetic induction in the air gap, T;

$\alpha_{\delta}$ - pole overlap coefficient;

$p$ - number of pairs of poles.

Sectional area of the yoke of the magnetic circuit of the electromagnetic element:

$$
S_{a}=\frac{\Phi_{\delta}}{2 B_{a}}
$$

The amplitude of the flow on the tine of the magnetic core of the electromagnetic element:

$$
\Phi_{z}=B_{\text {S.calc }} S_{t},
$$

The area of the end section of the tine of the magnetic circuit of the electromagnetic element:

$$
S_{z}=\frac{\Phi_{z}}{B_{z}},
$$

An important element of the calculation is determination of the parameters of the tine zone at a known total height of the magnetic circuit. From (7) and (8) the width of the groove of the magnetic circuit of the electromagnetic element: 


$$
b_{g}=\frac{\pi}{z}\left(1-\frac{B_{\delta . c a l c}}{B_{z}}\right) D_{m i d} .
$$

The cross-sectional area of the magnetic circuit groove of the electromagnetic element:

$$
S_{g}=\frac{\pi a A D_{\text {mid }}}{J k_{f} z} .
$$

where $a$-number of parallel branches;

$J$ - current density, A $\cdot \mathrm{m}^{-2}$;

$k_{f}$ - total filling factor of the slot of the magnetic circuit of the electromagnetic element.

Knowing the cross-sectional area of the groove, we find the height of the groove and the height of the yoke of the magnetic core of the electromagnetic element:

$$
\begin{gathered}
h_{g}=S_{g} / b_{g} . \\
h_{a}=S_{a} / l_{\delta} .
\end{gathered}
$$

The calculation of the winding data of the apparatus according to known methods [2;4] follows further.

\section{Results and discussion}

Two models of electromagnetic elements of the ferro-vortex apparatus with different parameters of the tine zone were constructed programmatically. In the first case (Fig. 2, a), the calculations were carried out taking into account (6), and in the second (Fig. 2, b) - (4) and (6). In this case, the ratio of the dimensions of the heights of the groove and yoke $\left(h_{g} / h_{a}\right)$, when calculating the magnetic core of the electromagnetic element of the FVA taking into account $l_{\text {d.calc }}$ is $7 / 3$, and when calculating without taking into account $l_{\text {..calc }}-4 / 6$. The cross-sectional area of the grooves of both magnetic cores and the number of turns in them were the same. The winding is double-layered.

a)

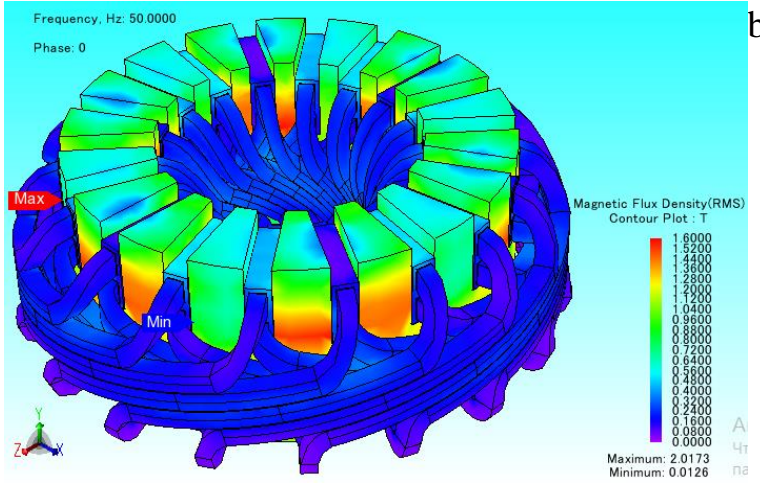

b)

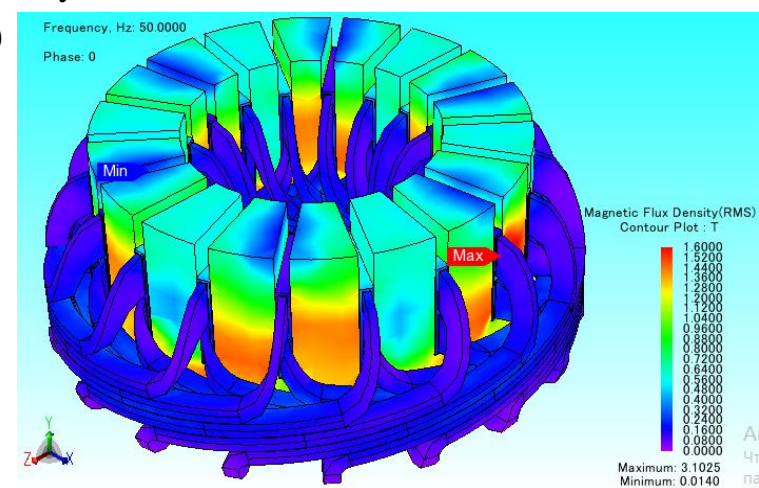

Fig. 2. Distribution of magnetic induction in magnetic circuits of electromagnetic elements: $\mathrm{a}-$ when calculating with $l_{\delta} ; \mathrm{b}-$ when calculating with $l_{\delta \text {.calc }}$

Further, the values of $B_{\delta}$ were measured programmatically in the section " $B$ " of the FVA working zone $[1 ; 11]$.

The values of $B_{\delta}$ were measured at control points (Fig. 3) at the surfaces of the magnetic core of the electromagnetic element and the axial magnetic core, incrementally increasing the distance between them. Since the axial magnetic core is made without grooves and winding, all control points were projected onto its surface from the surface of the tooth of the magnetic core of the electromagnetic element $[1 ; 11]$.

Based on the results of measurements in the MS Excel program, curves were constructed that limit the area of dependence of the magnetic induction in the gap $\left(B_{\delta}\right)$ on the value of $\delta$ [1] (Fig. $4 \mathrm{a}, \mathrm{b}$ ).

Using the MS Excel program for the trend lines of the average values of the magnetic induction in the working gap $\left(B_{\delta}\right)$ in the section " $B$ " of the working zone of the FVA, equations (Fig. 4 a, b) with a 
high level of approximation were obtained $\left(R^{2}=0.9819, R^{2}=0.9892\right)$, which indicates the adequacy of the mathematical description of these dependencies.

The analysis of the measurement results shows that the value of the magnetic induction in the working gap $\left(B_{\delta}\right)$ of the model constructed without taking into account the calculated active length of the electromagnetic element of the FVA $\left(l_{\text {s.calc }}\right)$ is significantly higher than with it. This can be explained by the fact that equation (6) must be applied only when calculating the parameters of magnetic cores of electromagnetic elements of the FVA with not yet known overall parameters $\left(D_{\text {out }}, D_{\text {in }}\right.$ and $\left.h=h_{a}+h_{z}\right)$. In both cases, there was a slight increase in the $B_{\delta}$ near the surface of the axial magnetowire model.

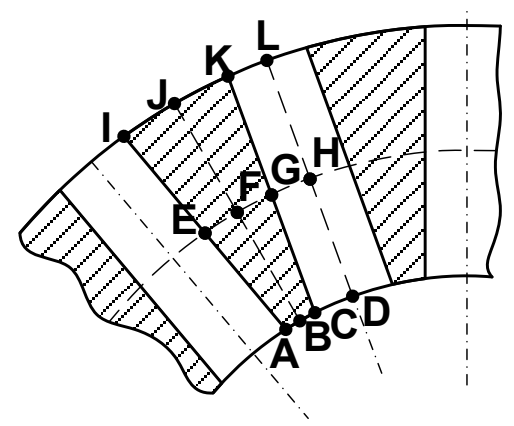

Fig. 3. Scheme of arrangement of control points

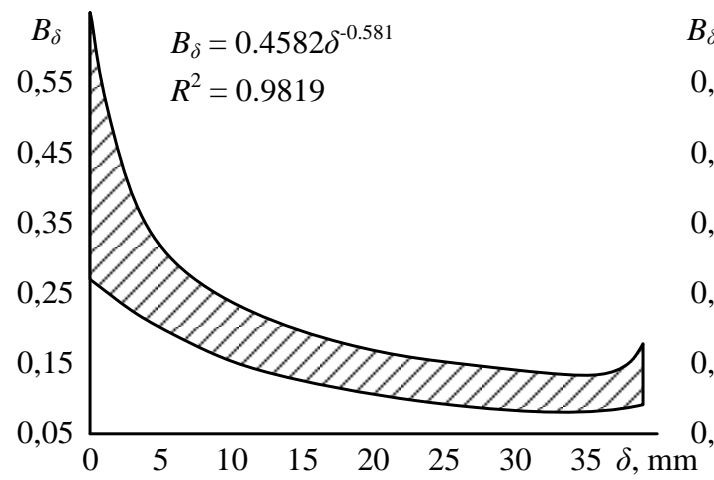

a)

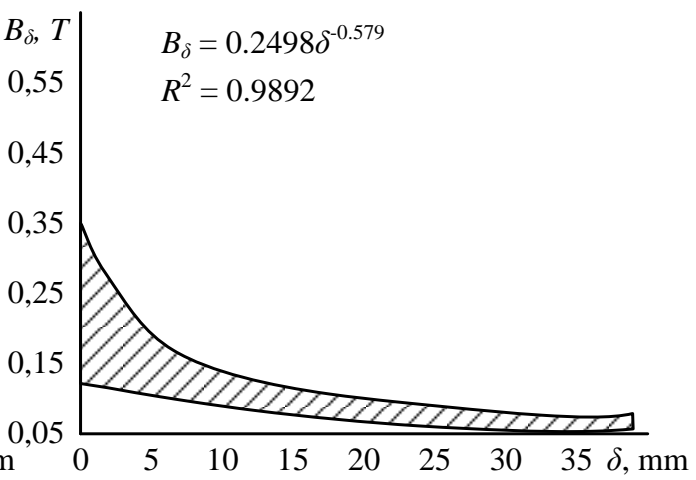

b)

Fig. 4. Dependence of $\boldsymbol{B}_{\delta}$ on $\delta$ at the control points of magnetic cores of electromagnetic elements: $\mathrm{a}$ - calculated with $l_{\delta} ; \mathrm{b}-$ calculated with $l_{\delta \text {.calc }}$

Studying the distribution pattern of magnetic induction over the volume of magnetic cores of electromagnetic elements, we drew attention to the fact that its maximum values in the magnetic core (Fig. 2, a) do not exceed the permissible values for this type of electrical steel. The magnetic core is magnetized evenly, except for the areas at the base of the tines. Significant saturation of steel is observed only in those tines, in the grooves between which the windings of the same phase are laid $(1,4,7,10$, $13,16)$. At the same time, individual areas remain unsaturated on the surface of the vertices of these tines.

In the magnetic circuit (Fig. 2, b) the picture of the magnetic field is more contrasting. The yoke is evenly saturated and significantly stronger than expected by calculations (2-2.5 times). The magnetization of the tines repeats the model (Fig. 2, a), but with great differences - the bases of the tines are more saturated with less saturated areas of the surface of their vertices.

\section{Conclusions}

1. The magnetic induction in the working gap $\left(B_{\delta}\right)$ of the model, built with known overall parameters ( $D_{\text {out }}, D_{\text {in }}$ and $h=h_{a}+h_{z}$ ), without taking into account the calculated active length of the electromagnetic element of the axial apparatus $\left(l_{\text {d.calc }}\right)$, is much higher than with it.

2. The calculated active length of the gap $\left(l_{\text {s.calc }}\right)$ should be used only when calculating the parameters of the magnetic circuits of the electromagnetic elements of the FVA with not yet known overall parameters $\left(D_{\text {out }}, D_{\text {in }}\right.$ И $\left.h=h_{a}+h_{z}\right)$. 
3. Based on the results of measurements of the values of magnetic induction $\left(B_{\delta}\right)$ in the section " $B$ " of the working zone of the FVA, curves were constructed that limit the area of dependence of the magnitude of magnetic induction in the gap $\left(B_{\delta}\right)$ on the size of the gap $(\delta)$.

4. When calculating the FVA, the value of the calculated magnetic induction in the working gap $\left(B_{\delta \text {.calc }}\right)$ should be used, equal to the ratio of the actual (required) induction in the gap $\left(B_{\delta \text {.fact }}\right)$ to the induction coefficient in the gap $\left(k_{B \delta}\right)$.

5. When analyzing the model, the optimal ranges of regulation of the size of the working zone (25$35 \mathrm{~mm})$ and the magnitude of the magnetic induction in the air gap (0.11-0.16 T) were determined.

\section{References}

[1] Adoshev A., Antonov S., Ivashina. A., Yastrebov S. Investigation of electromagnetic characteristics of ferro-vortex apparatus // Materials of the of 18th International Scientific Conference "Engineering for rural development". 2019. Volume 18. pp. 780-785.

[2] Игнатов В.А., Вильданов К.Я. Торцевые асинхронные электродвигатели интегрального изготовления (Butt-end asynchronous electric motors of the integral making). Moscow: Energoatomizdat, 1988. 301 p. (In Russian).

[3] Parviainen A. Design of axial-flux permanent-magnet low-speed machines and performance comparison between radial-flux and axial-flux machines: dis... doc. of science (technology): Lappeenranta, 2005. $153 \mathrm{p}$.

[4] Проектирование электрических машин: учебник для вузов/под ред. И.П. Копылова (Designing of electrical machines: textbook for universities). Moscow: High school, 2005. 767 p. (In Russian).

[5] Логвиненко Д.Д., Шеляков О.П. Интенсификация технологических процессов в аппаратах вихревого слоя (Intensification of technological processes in apparatus of the vortex layer). Kiev: Technique, 1976. $143 \mathrm{p}$.

[6] Tsantker K.L., Logvinenko D.D., Shelyakov O.P. etc. Apparatus for intermixing materials in a reaction vessel containing ferromagnetic particles. Patent (US) int. cl. B01j 1/00, No. 3869251. [online] [21.03.2021] Available at:

https://patents.google.com/patent/US3869251A/en?q= patent\%2fUS3869251A Recieved for publication May 14, 1973. Accepted after corrections March 4, 1975.

[7] Вершинин, Н.П. Установки активации процессов. «Использование в промышленности и в сельском хозяйстве. Экология» (The device of activation of processes. "A use in industry and in agriculture. Ecology"). Rostov-on-Don, 2004. 314 p. (In Russian).

[8] WIPO Patent Application WO/2007/114731 A1, B01J 19/12. Process activation unit. [online] [21.03.2021] Available at:

https://patentscope. wipo.int/search/ru/detail.jsf?docId = WO2007114731 Received for publication October 24, 2006. Accepted after corrections October 11, 2007.

[9] Wołosiewicz-Głab M. Construction of the electromagnetic mill with the grinding system, classification of crushed minerals and the control system/Wołosiewicz-Głab M., Ogonowski S., Foszcz D. // 17th IFAC Symposium on Control, Optimization and Automation in Mining, Mineral and Metal Processin. 2016, Volume 49, Issue 20. pp. 67-71.

[10] Ваганов М.А., Баранов Г.Д. Асинхронный торцевой двигатель с модифицированной геометрией зубцовой зоны статора (Asynchronous butt-end motor with modified geometry of the stator tooth zone). Izvestiya SPbGETU LETI. 2017. No. 2. pp. 48-53. (In Russian).

[11] Ferro-vortex apparatus/Adoshev A., Antonov S., Yastrebov S., Melnikov M. // Materials of the of 16th International Scientific Conference "Engineering for rural development", 2017. Volume 16. pp. 804-810. 